\title{
THEMIS/MSDP magnetic field measurements
}

\author{
A. Berlicki ${ }^{1,2}$, P. Mein $^{1}$, and B. Schmieder ${ }^{1,3}$ \\ 1 Observatoire de Paris, Section de Meudon, LESIA, 92195 Meudon Principal Cedex, France \\ e-mail: arkadiusz. berlicki@obspm. fr \\ 2 Astronomical Institute of the Wrocław University, ul. Kopernika 11, 51-622 Wrocław, Poland \\ 3 Institute of Theoretical Astrophysics, University of Oslo, Blindern, 0315 Oslo, Norway
}

Received 25 April 2005 / Accepted 11 July 2005

\section{ABSTRACT}

We present an analysis of longitudinal magnetic field measurements using the spectral data obtained with the French - Italian polarisation free telescope THEMIS working in Multichannel Subtractive Double Pass (MSDP) mode. We also used SOHO/MDI data to extend our analysis. THEMIS observations in the MSDP mode allows us to perform imaging spectropolarimetry characterized by high spatial and time resolution. In our analysis we used the observations of solar active region NOAA 10484 performed on October 20, 2003. From THEMIS 2D spectral images recorded in Na $D_{1} 5896 \AA$ line we obtained the longitudinal magnetic field in the active region. The value of the magnetic field was calculated at different distances from the $\mathrm{Na} D_{1}$ line centre. We determine the LOS magnetic field at different heights in the solar atmosphere. SOHO/MDI observations provide the longitudinal magnetic field in Ni I (6768 $\AA$ ) line. THEMIS/MSDP measurements at $\Delta \lambda=0.30 \AA$ are similar to SOHO/MDI results. Gradients of longitudinal magnetic fields derived from MSDP measurements at $\Delta \lambda=0.08$ and $\Delta \lambda=0.24 \AA$ exhibit different behaviours according to solar targets. A decrease with height is seen in spot umbrae and penumbrae, while the gradient in facular and network areas suggests a slight increase of the longitudinal magnetic field, which might be explained by the 3D-structure of canopies.

Key words. sunspots - Sun: magnetic fields - techniques: polarimetric

\section{Introduction}

The French-Italian THEMIS telescope operated since 1998 provides spectropolarimetric observations of different structures on the Sun. More details about THEMIS can be found at http: //www . themis.iac.es/. THEMIS is a polarizationfree telescope designed for spectropolarimetric observations of the Sun. In the Multichannel Subtractive Double Pass (MSDP) observing mode we are able to observe large fields-of-view with reasonable time resolution and high spatial resolution, reaching $0.5^{\prime \prime}$. Therefore, this instrument is useful for observing large active regions or filaments. Detailed description of the instrumental setup in the MDSP mode can be found in Mein (2002). In the MSDP mode THEMIS operates in two spectral lines simultaneously, depending on the requirements of the observer. For the whole field-of-view the two Stokes parameters ( $I$ and $V$ ) are obtained simultaneously for these chosen spectral lines. The intensity $(I)$ profile gives us the possibility to analyse the line profiles and the velocity field within solar structures, while the $V$ profile allows us to calculate the longitudinal component of the magnetic field. The velocity field can be derived from line asymmetries or shifts using the bisector method or more advanced methods based on the non-LTE radiative transfer modelling of the solar atmosphere (Heinzel 1995; Berlicki \& Heinzel 2004; Berlicki et al. 2005). Using I profiles we are also able to determine models of solar atmosphere. The longitudinal component of the magnetic field derived from $V$ Stokes profiles is useful in the analysis of the magnetic configuration of active regions and it can be used in magnetic field extrapolation procedures (Démoulin et al. 1997). Generally, during THEMIS observations we use one line which is magnetically sensitive and formed in the photosphere and low chromosphere (Na I lines). The second observed line is formed higher, in the solar chromosphere (H I or Ca II lines). In this situation, the first line is used mainly for magnetic field analysis, while the second one, for the velocity field and non-LTE radiative transfer modelling of the chromosphere.

The SOHO/MDI instrument (Scherrer et al. 1995; Liu et al. 2004) also permits us to obtain the longitudinal magnetic field but, in most cases, with a spatial resolution of about $2^{\prime \prime}$. In SOHO/MDI measurements, sets of five filtergrams in the Ni I $6768 \AA$ line (Landé factor $g=1.426$ ) with a sampling of $75 \mathrm{~m} \AA$ are used. The magnetic field is obtained from Doppler shifts measured separately in left and right circularly polarized light (Scherrer et al. 1995). The non-LTE calculations performed by Ding et al. (2002) showed that this line is formed at the heights close to the photosphere. Analysis of the contribution function obtained for this line by Ding et al. (2002) at different distances from the line centre allows us to conclude that the SOHO/MDI provides us the longitudinal magnetic field 
at the height of around $200 \mathrm{~km}$ above the photosphere (for the areas close to solar disk centre).

In the case of THEMIS/MSDP observations we are able to obtain the longitudinal magnetic field at different positions across the line profile. In other words, we get the longitudinal magnetic field at different heights in the solar atmosphere. Eibe et al. (2002) analysed the longitudinal magnetic field at three distances from the $\mathrm{Na} D_{1} 5896 \AA$ line centre $(0.080,0.160$ and $0.240 \AA$ ). Their analysis showed that the longitudinal component of the magnetic field changes with the height. In sunspot umbrae the magnetic field decreases with height, which can be interpreted as a result of the expansion of the magnetic flux tubes. In the network/plage areas the longitudinal component of the magnetic field seems to increase with height. Probably, the geometry of the magnetic field is dominant in this case.

Similar results have been obtained by Leka \& Metcalf (2003), by comparing the photospheric iron lines and $\mathrm{Na} D_{1}$ profiles at $\pm 68 \mathrm{~m} \AA$. In our paper, we use different positions in the $\mathrm{Na} D_{1}$ line profile, like Eibe et al. (2002), so that we observe exactly the same solar points at the same time. In addition, we include SOHO/MDI observations to compare the photospheric magnetic fields, as in Leka \& Metcalf (2003). The magnetic field measurements coming from the different types of solar structures is also discussed. In Sect. 2 we describe the data used in the analysis. The procedure of magnetic field determination and the results are given in Sect. 3. Section 4 contains the comparison of the THEMIS/MSDP and SOHO/MDI magnetic field data. Section 5 provides an analysis of magnetic field gradients obtained from THEMIS/MSDP data and the last section presents some final conclusions.

\section{Observations of the active region}

For our analysis we used the observations of the active region (AR) NOAA 10484 (Fig. 1). This AR belonged to the group of very active sunspot clusters observed in October/November 2003 (Zhang et al. 2003). These active regions produced many eruptive events including numerous X-class flares. The activity of NOAA 10484 has been presented in $\mathrm{Li}$ et al. (2005). On October 20, 2003 the AR was located at N03 E48, that corresponds to the angle between the lineof-sight and the line normal to the solar surface in the AR of about $44^{\circ}(\cos \theta=0.72)$.

The observations were obtained with THEMIS operating in the MSDP observing mode, during a coordinated observational campaign between ground based instruments (THEMIS at Tenerife and MISS multichannel spectrograph in Nanjing) and space observatories (SOHO/CDS and MDI, TRACE and RHESSI). The THEMIS/MSDP observations of the AR 10484 were performed simultaneously in two spectral lines, $5896 \AA \mathrm{Na} D_{1}$ and $8542 \AA \mathrm{CaII}$. The pixel size of the CCD used cameras corresponds to $0.2^{\prime \prime}$. The data obtained in $\mathrm{Na} D_{1}$ were used to analyse the longitudinal magnetic field.

The weather conditions allowed us to observe AR 10484 for many hours. We performed several scans covering a large field-of-view. The seeing conditions were best around 09-10 UT and these data are used in our analysis. In Fig. 2 we present the scan of the AR obtained between 09:13-09:32 UT,

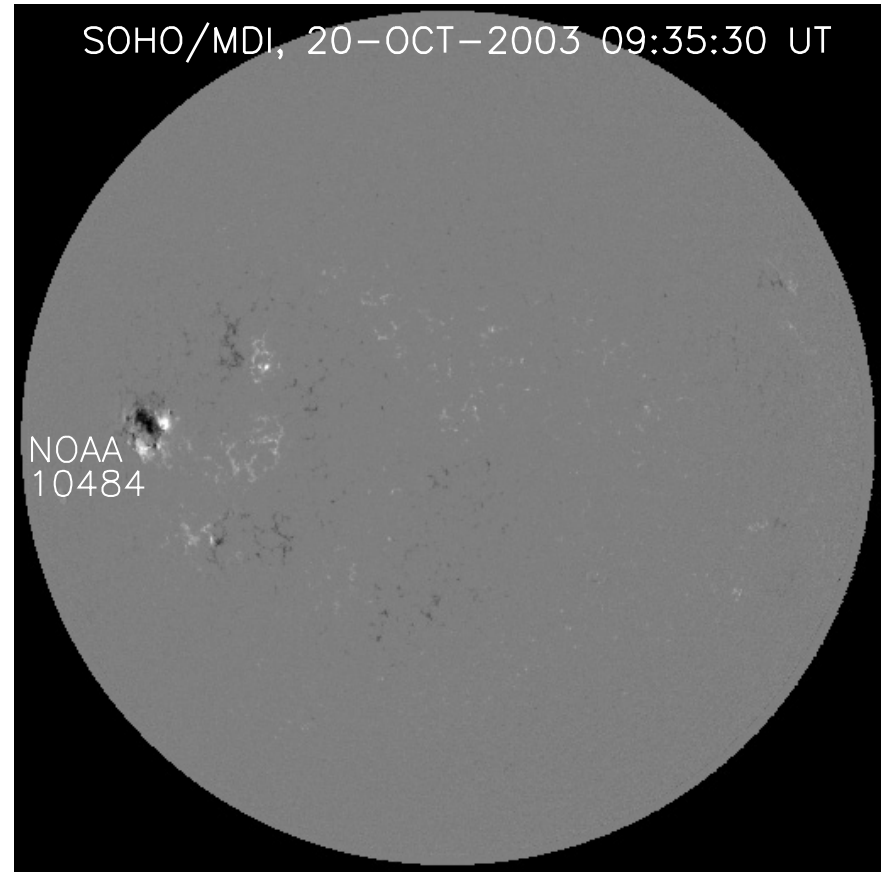

Fig. 1. SOHO/MDI full-disk magnetogram recorded on October 20, 2003. The heliographic coordinates of NOAA 10484 are N03 E48.

where the fine chromospheric structure is clearly visible, especially in the Ca II ( $8542 \AA$ ) line (lower panel). The upper panel presents the image taken in the $\mathrm{Na} D_{1}$ line which is also used for magnetic field determination.

\section{Magnetic field determined with THEMIS/MSDP and SOHO/MDI}

THEMIS/MSDP magnetic field measurements are derived from $I+V$ and $I-V$ line profiles observed simultaneously. We measure Zeeman shifts in each point of the 2D-field of view by a bisector method (Fig. 3).

For a given $\Delta \lambda$-value, we determine two points of equal intensity of each profile, so that the wavelength distance between both is equal to $2 \times \Delta \lambda$ ( $\mathrm{A} 1$ and $\mathrm{B} 1$ for $I+V, \mathrm{~A} 2$ and $\mathrm{B} 2$ for $I-V$ ). We compute intensities and wavelengths of middle points $(\mathrm{C} 1$ and $\mathrm{C} 2$ ). We call $\epsilon$ and $\delta \lambda$ the departures between values corresponding to $I+V$ and $I-V$ profiles. The longitudinal magnetic field $B_{\|}$(in Gauss) is derived from the relationship:

$\delta \lambda / 2=4.67 \times 10^{-13} g_{\mathrm{eff}} \lambda^{2} B_{\|}$

with $g_{\mathrm{eff}}=1.33$ for the $\mathrm{Na} D_{1}$ line. The wavelength $\lambda$ is given in Angstroms. $\epsilon$-values different from zero represent the accuracy of the overlap between $I+V$ and $I-V$ images. If the translation vector of the polarization analyser is not known accurately, it is possible to improve the value of this translation by minimizing $\epsilon$. Such a correction, possible with MSDP data, could not be done in both directions $x$ and $y$ in the case of usual 1D-spectroscopy.

In following sections, we present results obtained with THEMIS/MSDP between 9:13 and 9:32 UT. They are obtained for different values of $\Delta \lambda=0.08,0.16,0.24$ and $0.30 \AA$. The values 0.08 and 0.24 have been selected because they were 

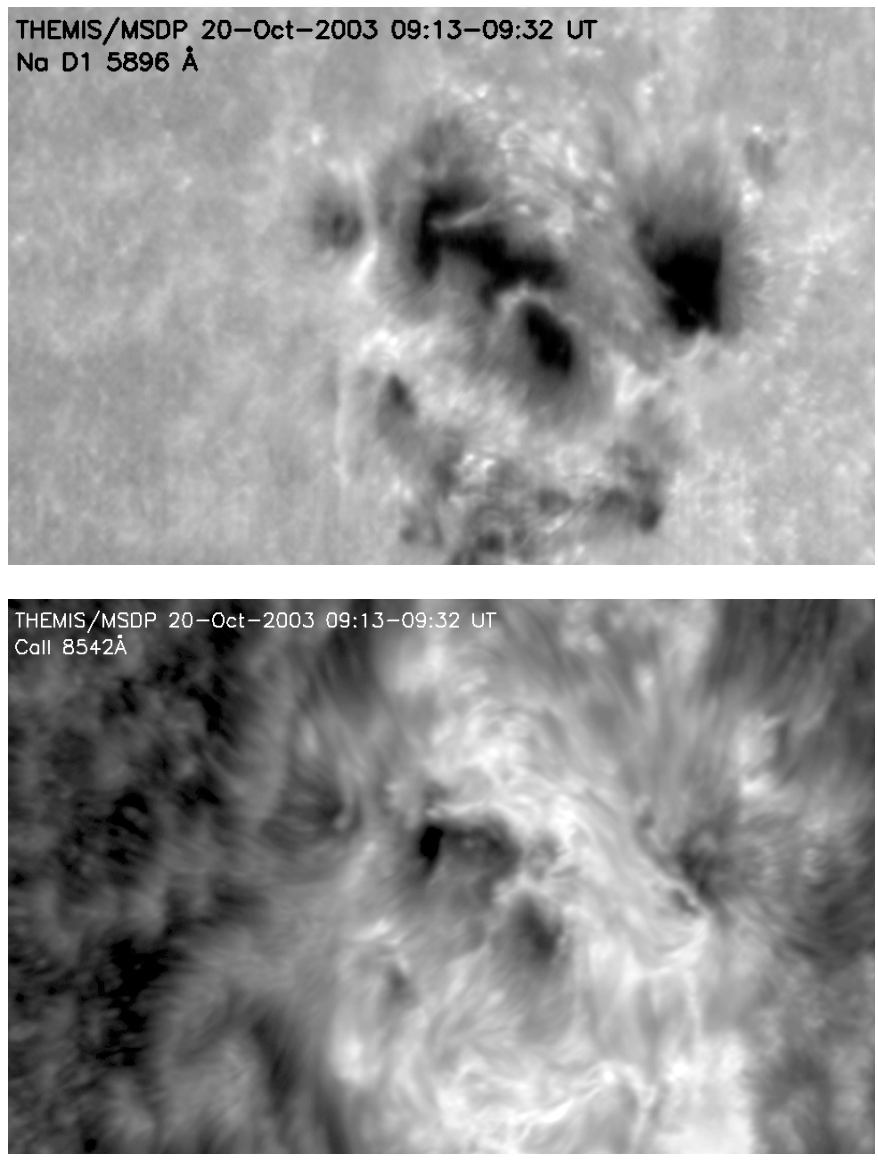

Fig. 2. THEMIS/MSDP images of the AR 10484 obtained on October 20, 2003 at 09:13-09:32 UT in the Na $D_{1} 5896 \AA$ (upper panel) and in Ca II 8542 A (lower panel) spectral lines.

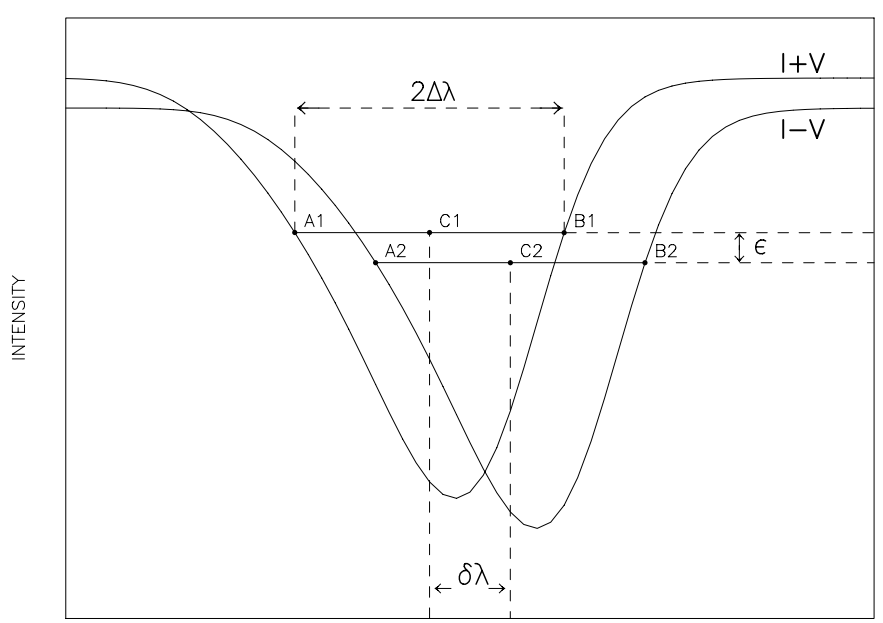

WAVELENGTH

Fig. 3. Profiles representing the method used for longitudinal magnetic field calculation. The Zeeman shifts $\Delta \lambda$ is deduced from bisector points $\mathrm{C} 1$ and $\mathrm{C} 2$.

also used in the paper by Eibe et al. (2002) for calculations of the height formation of $\mathrm{Na} D_{1}$ line using the response function analysis. The value $\Delta \lambda=0.30 \AA$ was selected in order to compare the magnetic field observed by SOHO/MDI and THEMIS/MSDP instruments (see next section).
The SOHO/MDI magnetic field map was extracted from the SOHO/MDI data base (http://sohowww nascom. nasa.gov/). We used the full-disk image obtained at 9:35 UT, closest to the time of THEMIS/MSDP scan. Due to the location of the NOAA 10484, only the low spatial resolution data (pixel of $2^{\prime \prime}$ ) are available.

As mentioned in Sect. 2 the considered active region NOAA 10484 was located rather far from the solar disk centre $(\cos \theta=0.72)$. In this situation the longitudinal component of the magnetic field that is measured by THEMIS does not correspond to the vertical component of the magnetic field.

\section{Comparison of THEMIS/MSDP and SOHO/MDI}

To make a comparative analysis of the magnetic field from THEMIS/MSDP and SOHO/MDI it was necessary to coalign both sets of data with high precision. First, in spite of different spatial resolutions, the map of SOHO/MDI was resized to get the same pixel-size as THEMIS/MSDP. The signal value was interpolated for the new pixels. Then, we coalign the THEMIS/MSDP with SOHO/MDI images using about 15 "control points" identified in THEMIS as well as in the MDI image. In Fig. 4 we present the coaligned images that were used for obtaining the cross-correlation (scatter) plots.

In order to disentangle contributions from spots and surrounding regions (Berger \& Lites 2003), we divide the field of view into two parts by using an intensity threshold. This intensity threshold was obtained empirically by using the THEMIS/MSDP intensity images in $\mathrm{Na} D_{1}$ line at $+0.3 \AA$ from the line centre. As indicated in Fig. 5, we select spot umbrae and penumbrae on one hand (left panel, hereafter "dark" regions), and facular and network areas on the other hand (right panel, hereafter "bright" regions). Limb darkening effects can be seen in the left part of the field-of-view in the case of "bright" regions.

The Fig. 6 shows pixel-to-pixel scatter plots of the longitudinal magnetic field observed by THEMIS/MSDP at $\Delta \lambda=$ $0.30 \AA$, compared to the SOHO/MDI magnetic field, in "dark" regions (left panel) and "bright" regions (right panel). Although the observations are obtained, neither at the same time, nor with the same spatial resolution, the correlation appears to be good. The slopes $Y$ of the regression lines are very close to $1(0.973$ and 1.002 respectively), which shows that the formation altitudes of $\mathrm{Na} D_{1}$ at $0.3 \AA$ and Ni I lines are very similar. This is consistent with the $200 \mathrm{~km}$ altitude assumed for the Ni I line (Ding et al. 2002) used for SOHO/MDI measurements at disc centre. Indeed, the formation altitude computed by Eibe et al. (2002) for $\mathrm{Na} D_{1}$ at $\Delta \lambda=0.24 \AA$ lies between 180 (Maltby umbra model) and $280 \mathrm{~km}$ (VAL-C model). These altitudes are measured along the vertical line-of-sight. The constant departures $X$ are inside the expected accuracy range (less than $20 \mathrm{G}$ ). The value of the longitudinal magnetic field in "bright" regions is contained within $\pm 500 \mathrm{G}$, while in "dark" regions (sunspots) we observe a magnetic field larger than $1200 \mathrm{G}$. The standard deviation $\sigma$ of $X$ and $Y$ parameters obtained for least-square linear fits are given in the plots (Fig. 6). The deviation of $Y$ parameters from 1.00 is not significant for "bright" areas (right panel), but in the case of spot umbrae and penumbrae (left 

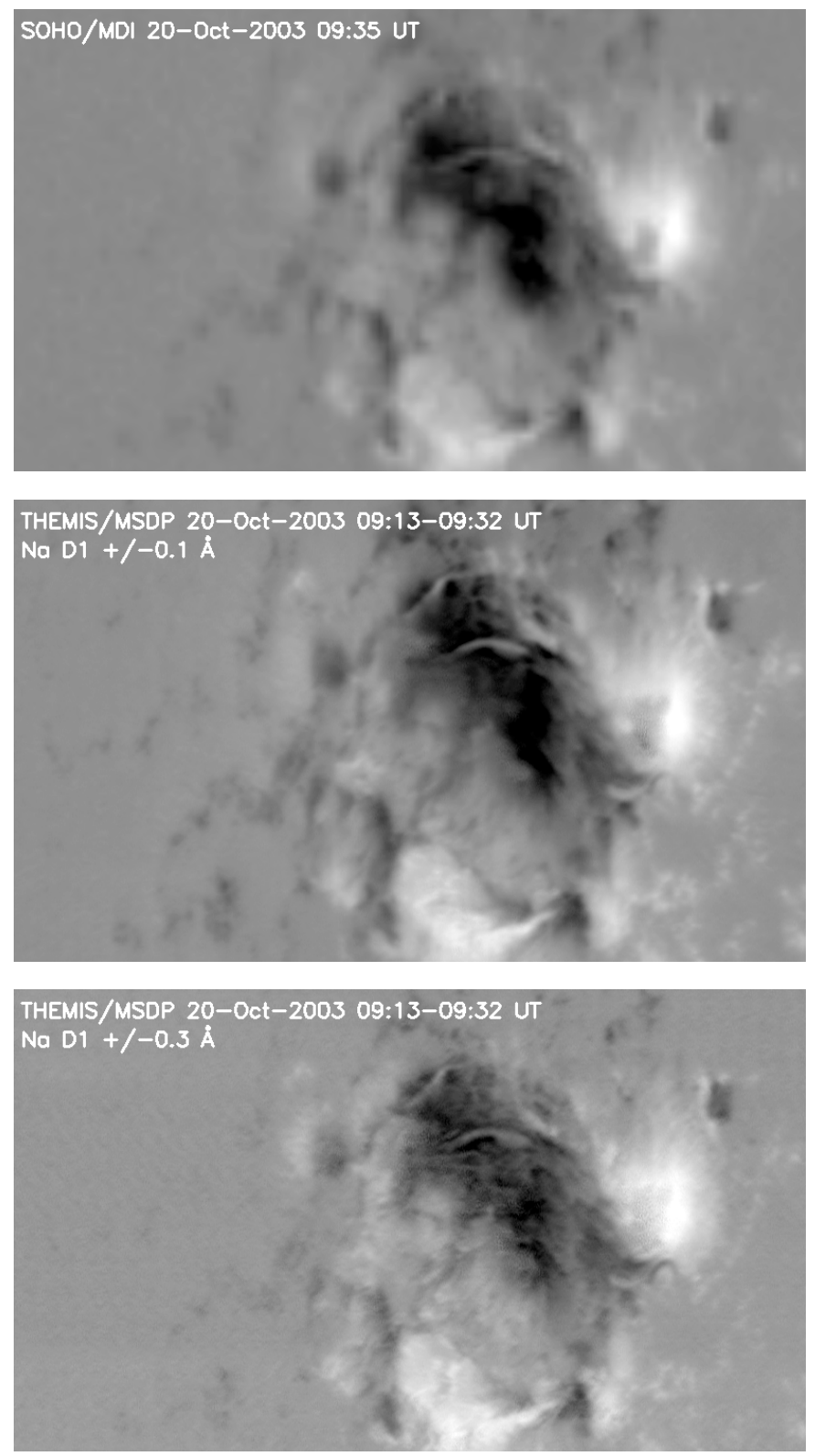

Fig. 4. Coaligned images of the longitudinal magnetic field of the AR 10484 obtained on October 20, 2003 with SOHO/MDI (upper panel) and with THEMIS/MSDP (middle and lower panels). SOHO/MDI magnetic field was determined using Ni I 6768 Å photospheric line while in THEMIS/MSDP observations the Na $D_{1} 5896 \AA$ chromospheric line was used. The field-of-view of all images is $206^{\prime \prime} \times 120^{\prime \prime}$.

panel), the lower than 1.00 value of the $Y$ parameter suggests that the average altitude of $\mathrm{Ni}$ I line formation may be a little lower than the formation altitude of the $\mathrm{Na} D_{1}$ line at $0.3 \AA$.

The similarity of results towards high values $(1000 \mathrm{G})$ shows that the saturation effects are not very different, although they are not due to the same effects. THEMIS/MSDP data are mainly affected by scattered light, although both successive gratings of THEMIS strongly reduce this effect, at least for wavelength analysis. SOHO/MDI data, which do not use a bisector method, are more sensitive to non-linear effects at large Zeeman shifts.

\section{Gradient of longitudinal magnetic field from THEMIS/MSDP data}

Scatter plots of THEMIS/MSDP data at two $\Delta \lambda$-values are shown in Fig. 7, for "dark" and "bright" regions defined previously. Abscissae correspond to $0.24 \AA$ and ordinates to $0.08 \AA$. Stray light effects are partly compensated for the magnetic field ratios.

Our data were processed in the same way as in the paper by Eibe et al. (2002), where according to the theoretical results, the expected difference between formation altitudes of $\mathrm{Na} D_{1}$ line at 0.24 and $0.08 \AA$ from the line centre lies roughly between $200 \mathrm{~km}$ (Maltby model) and $250 \mathrm{~km}$ (VAL-C model) (see Table 1 of the same paper). Although our observations are far from disc centre, we assume that the altitude difference is similar, close to $225 \mathrm{~km}$.

In the "dark" regions, (Fig. 7 - left panel), the longitudinal magnetic field is generally decreasing with height, because of the vertical expansion of lines of force. The slope of the regression line is smaller than 1 . The relative decrease is around $L^{-1}=-6.2 \times 10^{-4} \mathrm{~km}^{-1} . L^{-1}$ is gradient of the absolute longitudinal magnetic field along the line-of-sight $l$, defined as:

$L^{-1}=\frac{1}{B_{\|}(l=0)}\left(\frac{\partial B_{\|}}{\partial l}\right)$

On the contrary, for "bright" regions (Fig. 7 - right panel) the longitudinal magnetic field appears to increase slightly with height for small values, leading to a slope of the regression line larger than 1 , and to decrease for higher values, leading to a S-shape of the scatter plot. We may suggest qualitatively the following interpretations. In areas where canopies are present, two cases may be considered:

a) very small magnetic fields: lines-of-sight may successively cross zero magnetic field layers (below canopies) and small magnetic field layers (inside canopies);

b) medium values of magnetic field (ends of the S-shape): they may correspond to regions of concentrated flux with opening lines of force at canopy feet.

Figure 8 presents the areas where the longitudinal magnetic field gradient derived from both THEMIS/MSDP determinations is significant. The behaviour of "dark" and "bright" areas can be seen in more details. In spite of strong perspective effects due to the large distance from disc centre, the general trend is shown qualitatively, but the position of sunspots is shifted with respect to the areas with negative gradients of the magnetic field. In our analysis we did not find any clear correlation between the derived magnetic field gradient and the line core widths. Therefore, the non-thermal velocity and the effects of optical depth variations probably have only minor influence on our results.

\section{Conclusions}

We coaligned data from THEMIS/MSDP and SOHO/MDI obtained on October 20, 2003. MSDP magnetic fields are derived from a bisector method at different positions in the $\mathrm{Na} D_{1}$ profile. 

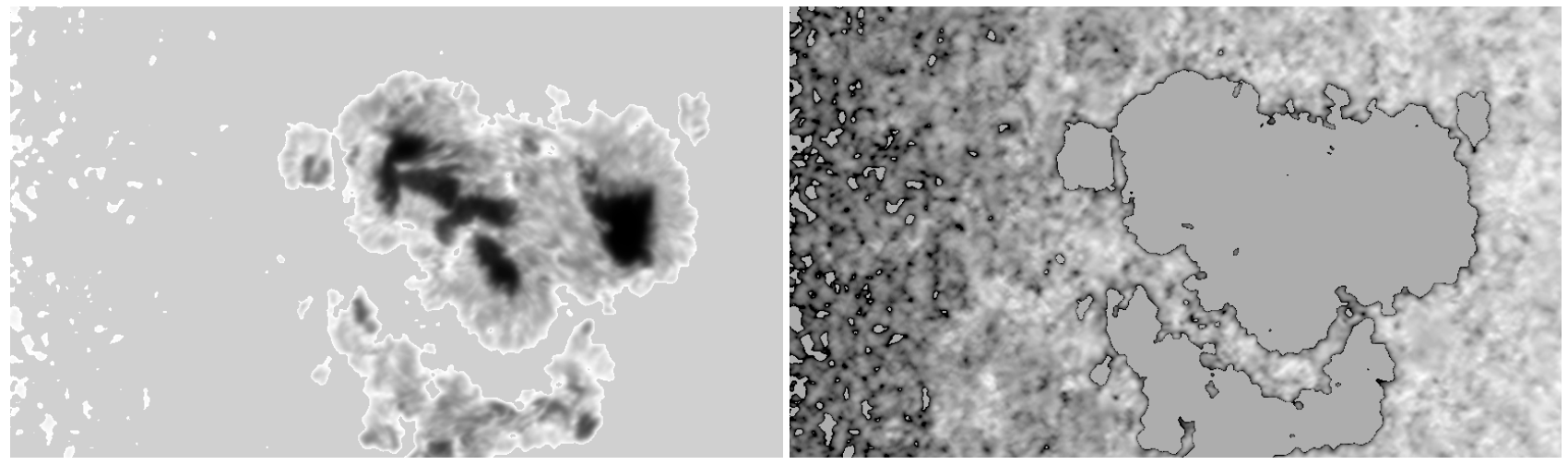

Fig. 5. THEMIS/MSDP images of the AR 10484 at $+0.3 \AA$ from the Na $D_{1}$ line centre. With the grey colour we marked the areas not used for the analysis of the scatter plots. In the left panel the analysed area corresponds to the sunspots umbrae and penumbrae ("dark" regions), while the right panel presents the area covering the network ("bright" regions).
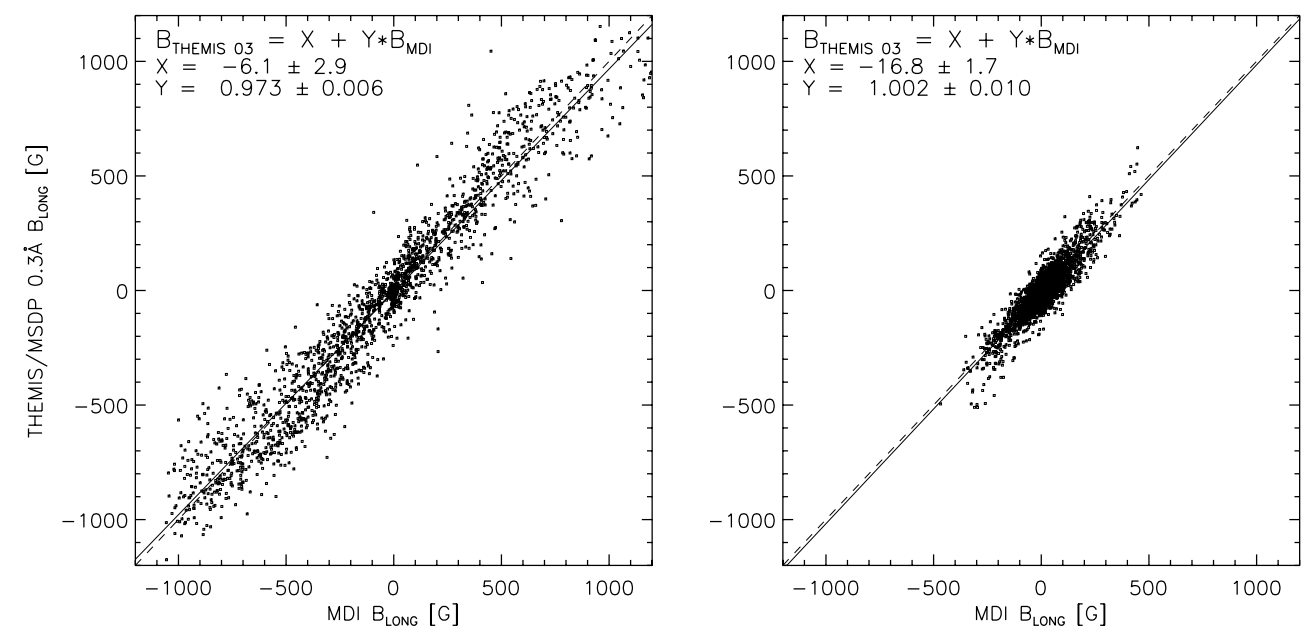

Fig. 6. Scatter plots of the longitudinal magnetic field $B_{\text {THEMIS }}$ at $0.3 \AA$ vs. $B_{\mathrm{MDI}}$, plotted only for the area covering the sunspots - "dark" regions (left panel) and for the area covering the solar network - "bright" regions (right panel). Dashed line represents the equal-value line while continuous line shows the linear fit obtained for these two sets (THEMIS and MDI) of data. $X$ and $Y$ are the parameters of linear fit.
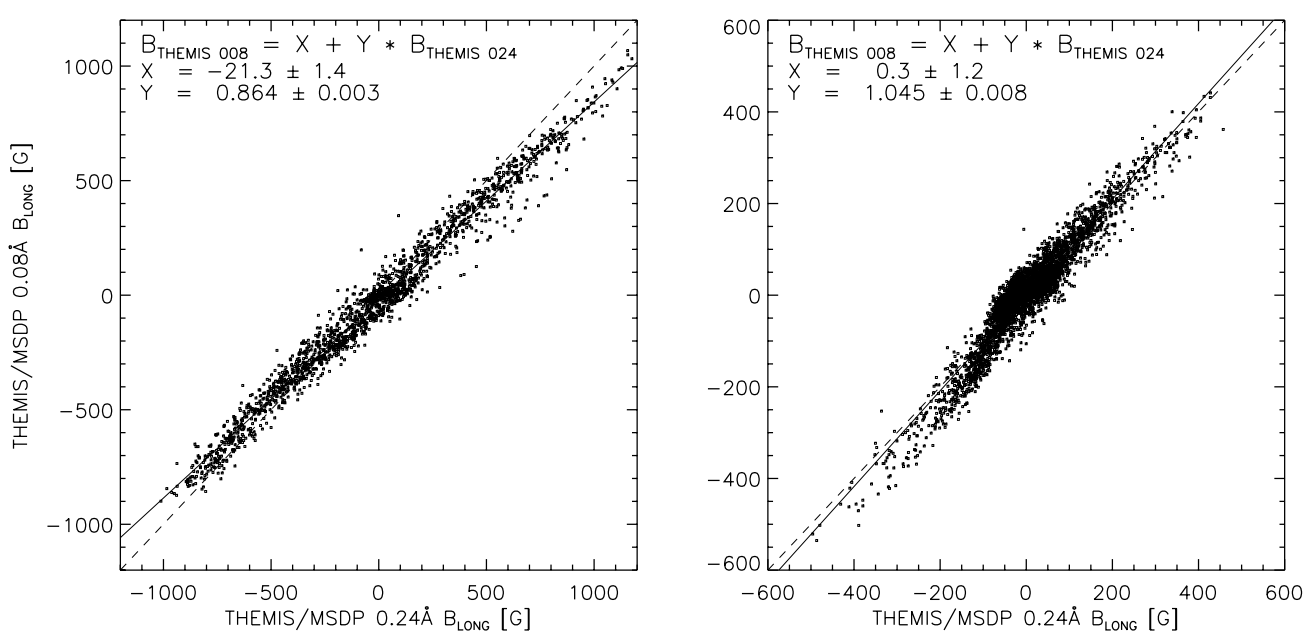

Fig. 7. Scatter plots of the longitudinal magnetic field $B_{\text {THEMIS }}$ at $0.08 \AA$ vs. $B_{\text {THEMIS }}$ at $0.24 \AA$, plotted only for the area covering the sunspots (left panel) and for the area covering only the solar network (right panel). The dashed line represents the equal-value line. The continuous line is the linear fit obtained for these two sets of data. $X$ and $Y$ are the parameters of this fit. Note that in the right panel the axis ranges are restricted to $\pm 600 \mathrm{G}$ 


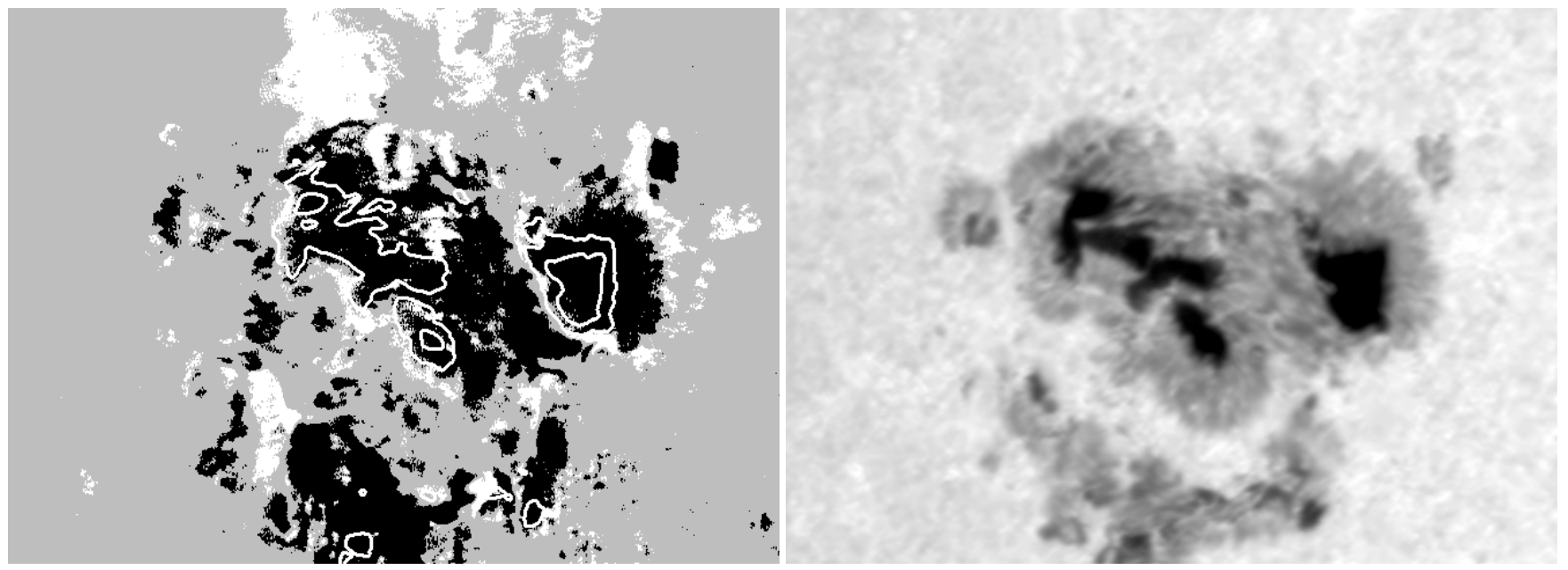

Fig. 8. THEMIS/MSDP image of the longitudinal magnetic field gradient (left panel) obtained from magnetic field maps in Na $D_{1}$ line at $0.08 \AA$ and $0.24 \AA$. White: increase, black: decrease of magnetic field with height; grey: no significant gradient of magnetic field found. The contours mark the sunspot umbrae. For comparison, in the right panel we present the image in Na $D_{1}$ line at $+0.24 \AA$ from the line centre.

The similarity of MDI results to MSDP values computed at $\Delta \lambda=0.3 \AA$ suggest that the formation altitude of the Ni I $6768 \AA$ line is close to the formation altitude of the $\mathrm{Na} D_{1}$ line at $0.3 \AA$ from line centre, as far as Zeeman shifts are concerned.

The comparison of THEMIS/MSDP data at $\Delta \lambda=0.08 \AA$ and $\Delta \lambda=0.24 \AA$ shows that:

- Umbrae and penumbrae of spots exhibit longitudinal magnetic fields decreasing with height, as already shown in Eibe et al. (2002) and Leka \& Metcalf (2003).

- Low field areas of faculae and network suggest increases of the longitudinal magnetic field with height.

We propose tentative explanations, taking into account the opening of lines of force and the geometry of canopies. More data would be necessary to understand this geometry. High spatial resolution allowing a fine comparison of magnetic structures along the $\mathrm{Na} D_{1}$ profile would be very helpful in this respect. To improve the quality of the analysis it also would be better to observe some active regions located closer to the solar disk centre. Better calculations of the response function for Na I, Ni I and other lines used for the magnetic field analysis are also necessary. Then, the scatter plots of the longitudinal magnetic field would be less uncertain.

Acknowledgements. This research was supported by the European Commission through the RTN programme ESMN (European Solar
Magnetism Network, contract HPRN-CT-2002-00313). The authors are members of ESMN. We would like to thank the THEMIS team who operates the telescope. THEMIS is French-Italian telescope operated on the island of Tenerife by CNRS-CNR in the Spanish Observatorio del Teide of the Instituto de Astrofisica de Canarias. $\mathrm{SOHO}$ is a joint project between ESA and NASA.

\section{References}

Berger, T. E., \& Lites, B. W. 2003, Sol. Phys., 213, 213

Berlicki, A., \& Heinzel, P. 2004, A\&A, 420, 319

Berlicki, A., Heinzel, P., Schmieder, B., Mein, P., \& Mein, N. 2005, A\&A, 430, 679

Démoulin, P., Bagala, L. G., Mandrini, C. H., Hénoux, J. C., \& Rovira, M. G. 1997, A\&A, 325, 305

Ding, M. D., Qiu, J., \& Wang, H. 2002, ApJ, 576, L83

Eibe, M. T., Aulanier, G., Faurobert, M., Mein, P., \& Malherbe, J. M. 2002, A\&A, 381, 290

Heinzel, P. 1995, A\&A, 299, 563

Leka, K. D., \& Metcalf, T. R. 2003, Sol. Phys., 212, 361

Li, H., Berlicki, A., \& Schmieder, B. 2005, A\&A, 438, 325

Liu, Y., Zhao, X., \& Hoeksema, J. T. 2004, Sol. Phys., 219, 39

Mein, P. 2002, A\&A, 381, 271

Scherrer, P. H., Bogart, R. S., Bush, R. I., et al. 1995, Sol. Phys., 162, 129

Zhang, H. Q., Bao, X. M., Zhang, Y., et al. 2003, Chin. J. Astron. Astrophys., 3, 6, 491 